\title{
Giffen Goods and Market Making *
}

\author{
Giovanni Cespa ${ }^{\dagger}$
}

First draft: April, 2002

This draft: May, 2003

\begin{abstract}
This paper shows that information effects per se are not responsible for the Giffen goods anomaly affecting competitive traders' demands in multi-asset, noisy rational expectations equilibrium models. The role that information plays in traders' strategies also matters. In a market with risk averse, uninformed traders, informed agents have a dual motive for trading: speculation and market making. While speculation entails using prices to assess the effect of private signal error terms, market making requires employing them to disentangle noise traders' effects in traders' aggregate orders. In a correlated environment, this complicates a trader's signal-extraction problem and may generate upward-sloping demand curves. Assuming either (i) that competitive, risk neutral market makers price the assets, or that (ii) the risk tolerance coefficient of uninformed traders grows without bound, removes the market making component from informed traders' demands, rendering them well behaved in prices.
\end{abstract}

Keywords: Financial Economics-Asset Pricing-Information and Market Efficiency. JEL Classification: G100, G120, G140.

${ }^{*}$ I thank Anat Admati, Jordi Caballé, Giacinta Cestone and Xavier Vives for useful suggestions. The comments provided by the associate editor and an anonymous referee greatly improved the paper's exposition. Hospitality and financial support from the Ente per gli Studi Monetari e Finanziari "Luigi Einaudi," are gratefully acknowledged.

${ }^{\dagger}$ Departament d'Economia i Empresa, Universitat Pompeu Fabra, Ramon Trias Fargas, 25-27, E-08005, Barcelona. E-mail: giovanni.cespa@econ.upf.es. Tel. +34 (93) 54227 08. Fax +34 (93) 5421746. 


\section{Introduction}

In a well known paper, Admati (1985) showed that multi-asset, noisy rational expectations equilibrium (NREE) markets display a number of anomalies. In particular, owing to correlation effects, traders' demand functions could be upward-sloping in prices. This "Giffen goods" anomaly was attributed to the contemporaneous workings of an information and a substitution effect generated by prices in an economy with asymmetric information. Indeed, a price increase in a NREE could either signal an increase in the value of the asset pay-off or be the effect of a demand pressure from noise traders. For some parameter configurations, traders could then interpret a price increase as good news about the asset's fundamental and increase their (long) position in the asset. Recently, Giffen goods anomalies have been related to market behavior around "crashes" (see Gennotte and Leland (1990) and Barlevy and Veronesi (2002)), to "market breakdowns" (see Battacharya and Spiegel (1991)) and to unstable equilibria (see Cespa (2002)). Upward sloping demand curves make traders shy away from assets whose price plummets and increase their long position in assets whose price rockets, eventually amplifying market movements or preventing trade from happening at all in equilibrium. ${ }^{1}$ In this perspective, understanding the extent to which information effects per se determine such anomalies is therefore relevant.

This paper shows that information effects alone are not responsible for Giffen goods anomalies: the role that information plays in traders' strategies also matters. Intuitively, privately informed traders should be able to better disentangle noise from information and this should lead them to choose their positions by comparing prices with their private signals. On the contrary, traders that only observe (endogenous) public information (i.e. equilibrium prices) should rely on correlation effects in order to disentangle the informative content of a price movement. Building on this insight, I show that in a market with risk-averse, uninformed traders, informed agents have a dual motive for trading: speculation and market making. They speculate on the difference between their private signals and equilibrium prices; they accomodate traders' total demand in each asset by comparing (common) prior information to equilibrium prices. While speculation entails assessing the effect of private signal biases, market making requires disentangling noise traders' effects from fundamental information within the observed aggregate orders. The latter complicates the signal extraction problem and

\footnotetext{
${ }^{1}$ In the Bhattacharya and Spiegel's model, a market breakdown occurs whenever the "uninformed" agents estimate that the insider's informational trading motive overcomes his hedging motive. As a result, instead of taking the other side of the order, they align their trades to those of the insider (and this, in the linear equilibrium case, potentially generates the Giffen anomaly). However, owing to the lack of "noise" traders, no one absorbs the aggregate order, and a breakdown ensues.
} 
(may) generate upward sloping demand curves. I therefore attribute Admati's "Giffen" goods anomaly to the market making component of informed traders' demands.

Based on this intuition, I then give sufficient conditions under which the Giffen goods phenomenon disappears from informed traders' strategies. Intuitively, this occurs whenever informed traders find it unprofitable to accomodate liquidity shocks. Thus, either assuming that competitive, risk neutral market makers price the assets or letting the risk tolerance parameter of uninformed traders grow unboundedly, allows to remove the anomaly from the demand of informed agents but not from that of uninformed agents.

The paper is organized as follows: in the next section I outline the model's assumptions, define notation and recall the equilibrium result of Admati (1985). I then show by means of examples that the market making component of an informed trader's demand is responsible for the Giffen goods anomaly. In section 3 I introduce risk neutral competitive market makers in the model and show that this removes the anomaly from informed demands. In section $4 \mathrm{I}$ generalize the model in two ways. First, I consider a market where informed and uninformed traders interact. This allows to show that the result of section 3 can be obtained as a limit result when uninformed traders' risk tolerance grows unboundedly. Next, I show that the result obtained in the current simplified version of Admati (1985) applies to the equilibrium found in her paper as well.

\section{The Model}

In this section I consider a simplified version of the market studied by Admati (1985). Two classes of agents exchange a vector of $K$ risky assets with random liquidation value $\boldsymbol{v} \sim N\left(\overline{\boldsymbol{v}}, \boldsymbol{\Pi}_{\boldsymbol{v}}^{-1}\right)$ and a riskless one with unitary return: a continuum of risk-averse informed traders distributed in the interval $[0,1]$ and noise traders, trading for liquidity purposes. Each informed agent $i$ receives a $K$-dimensional vector of private signals $\boldsymbol{s}_{i}=\boldsymbol{v}+\boldsymbol{\epsilon}_{i}$ where $\boldsymbol{\epsilon}_{i} \sim N\left(\mathbf{0}, \boldsymbol{\Pi}_{\boldsymbol{\epsilon}}^{-1}\right)$, and $\boldsymbol{\epsilon}_{i}, \boldsymbol{\epsilon}_{j}$ are independent for $i \neq j$. Assume that his preferences are represented by a CARA utility $U\left(\pi_{i}\right)=-\exp \left\{-\pi_{i} / \gamma\right\}$ where $\gamma>0$ is the coefficient of constant absolute risk tolerance and $\pi_{i}=\boldsymbol{x}_{i}^{\prime}(\boldsymbol{v}-\boldsymbol{p})$ is the profit of buying $\boldsymbol{x}_{i}^{\prime}=\left(x_{i 1}, x_{i 2}, \ldots, x_{i K}\right)$ units of each asset at price $\boldsymbol{p}$. Normalize the informed traders' initial wealth to zero and let noise traders submit a $K$-dimensional vector of random demands $\boldsymbol{u} \sim N\left(\mathbf{0}, \boldsymbol{\Pi}_{\boldsymbol{u}}^{-1}\right)$. Assume that the random vectors $\boldsymbol{v}, \boldsymbol{u}, \boldsymbol{\epsilon}_{i}$ are independent $\forall i$ and that the Strong Law of Large Numbers holds (i.e. $\int_{0}^{1} \boldsymbol{\epsilon}_{i} d i=\mathbf{0}$, almost surely). Finally, let each of $\boldsymbol{\Pi}_{\boldsymbol{v}}^{-1}, \boldsymbol{\Pi}_{\boldsymbol{u}}^{-1}$, and $\boldsymbol{\Pi}_{\boldsymbol{\epsilon}}^{-1}$ be positive definite and suppose that the distributional assumptions are common knowledge among the agents in 
the economy. 2

\subsection{The Equilibrium}

Suppose that in the above market each trader submits a vector of demand functions indicating the position desired in each asset at every price, contingent on his private information. Owing to market clearing, the resulting equilibrium price vector will then reflect all traders' information. This, in turn, will provide each agent with an additional signal beyond the one he privately observes, that he can exploit in forming his optimal demand. Therefore, in a rational expectations equilibrium, prices perform two functions: they clear all markets and they convey information to traders. In turn, traders' beliefs are endogenous and their demand functions are defined only for equilibrium prices. ${ }^{3}$

The following definition formally describes the rational expectations equilibrium concept for the above market:

Definition 1 A rational expectations equilibrium for the above market is a price vector $\boldsymbol{p}$ and demand functions $\left\{\mathbf{X}_{i}\left(\boldsymbol{s}_{i}, \boldsymbol{p}\right)\right\}_{i \in[0,1]}$ such that (i) $\boldsymbol{p}$ is $(\boldsymbol{v}, \boldsymbol{u})$ measurable; (ii) $\mathbf{X}_{i}\left(\boldsymbol{s}_{i}, \boldsymbol{p}\right) \in$ $\arg \max \boldsymbol{x}_{i} E\left[U\left(\pi_{i}\right) \mid \boldsymbol{s}_{i}, \boldsymbol{p}\right]$; (iii) $\int_{0}^{1} \mathbf{X}_{i}\left(\boldsymbol{s}_{i}, \boldsymbol{p}\right) d i+\boldsymbol{u}=0$ almost surely.

The first condition requires prices not to depend on single signals' realizations. Indeed, in the large market as each informed agent is small and private signals are independently distributed, equilibrium prices should only vary either because of changes in the value of the pay-off vector or because of noise traders' demand realizations. The second condition requires traders to choose optimal equilibrium demand functions given the equilibrium price and their private information. Finally, the last condition requires the price vector to clear all the markets.

To apply definition 1 to the current context, assume each informed trader $i$ submits a vector of demand functions $\mathbf{X}_{i}\left(\boldsymbol{s}_{i}, \boldsymbol{p}\right)$ and restrict attention to equilibria where the price is a linear function of informed traders' aggregate signals and noise traders' demands. Owing to CARA utility and the normality assumption, an informed agent's equilibrium demand is then linear in his private signal $\boldsymbol{s}_{i}$ and in the equilibrium price vector $\boldsymbol{p}$. Indicate with $\mathbf{X}_{i}\left(\boldsymbol{s}_{i}, \boldsymbol{p}\right)=\mathbf{A} \boldsymbol{s}_{i}+\phi(\boldsymbol{p})$ a candidate vector of equilibrium demand functions, where $\mathbf{A}$ and $\phi(\boldsymbol{p})$ are respectively the matrix of trading aggressiveness and a linear function of the price to be

\footnotetext{
${ }^{2}$ It is worth stressing that informed agents receive i.i.d. private signals. This assumption allows to simplify the equilibrium closed form solution with respect to Admati (1985), and is made without loss of generality, since, as I will show in section 4.2, relaxing it does not change the results of the paper.

${ }^{3}$ For noisy rational expectations equilibrium models with a single risky asset see Hellwig (1980), Diamond and Verrecchia (1981) and Grossman and Stiglitz (1980).
} 
determined in equilibrium. The market clearing equation thus reads as $\int_{0}^{1} \mathbf{A} \boldsymbol{s}_{i} d i+\phi(\boldsymbol{p})+\boldsymbol{u}=\mathbf{0}$, and the following result holds:

Proposition 1 In the market outlined above there exists a unique linear equilibrium where agents' strategies and the equilibrium price vector are given by:

$$
\begin{aligned}
\mathbf{X}_{i}\left(\boldsymbol{s}_{i}, \boldsymbol{p}\right) & =\mathbf{A}\left(\boldsymbol{s}_{i}-\boldsymbol{p}\right)+\left(\boldsymbol{\Lambda}^{-1}-\mathbf{A}\right)(\overline{\boldsymbol{v}}-\boldsymbol{p}) \\
\boldsymbol{p} & =\boldsymbol{\Lambda} \boldsymbol{z}+(I-\boldsymbol{\Lambda} \mathbf{A}) \overline{\boldsymbol{v}}
\end{aligned}
$$

where $\mathbf{A}=\gamma \boldsymbol{\Pi}_{\boldsymbol{\epsilon}}, \boldsymbol{z}=\mathbf{A} \boldsymbol{v}+\boldsymbol{u}, \boldsymbol{\Lambda}=(\mathbf{A}+\gamma \boldsymbol{\Pi})^{-1}(I+\gamma \mathbf{A} \boldsymbol{\Pi} \boldsymbol{u})$, and $\boldsymbol{\Pi}=(\operatorname{Var}[\boldsymbol{v} \mid \boldsymbol{z}])^{-1}=$ $\Pi_{v}+\mathrm{A}_{\boldsymbol{u}} \mathrm{A}$.

Proof. See Admati (1985).

QED

The vector $\boldsymbol{z}$ measures the total net demand due to traders' private information and noise traders' supply shocks. Insofar as it conveys a signal about the "true" value of the asset payoffs, it captures the "informational content" of the order flows. The matrix $\Lambda^{-1}$ maps equilibrium prices into the traders' total net demand: for a unitary price vector $\boldsymbol{p}^{\prime}=(1,1, \ldots, 1), \boldsymbol{\Lambda}^{-1}$ measures the size of the traders' aggregate demand in each asset that is either due to private information or to a liquidity shock.

According to (2.1) an informed agent's demand function has two components. The first component $\left(\mathbf{A}\left(\boldsymbol{s}_{i}-\boldsymbol{p}\right)\right)$ is proportional to the difference between the vector of private signals and the vector of equilibrium prices. It reflects the agent's "speculative" position based on his private information. The second component $\left(\left(\boldsymbol{\Lambda}^{-1}-\mathbf{A}\right)(\overline{\boldsymbol{v}}-\boldsymbol{p})\right)$ is proportional to the difference between the vector of assets' unconditional expectations and the vector of equilibrium prices. This component reflects the investor's position in (potentially) accommodating the total net demand in each asset $k$.

A trader's speculative aggressiveness is given by the conditional precision matrix of his private signals weighted by his risk tolerance coefficient: $\mathbf{A}=\gamma \boldsymbol{\Pi}_{\boldsymbol{\epsilon}}$. As $\boldsymbol{\Pi}_{\boldsymbol{\epsilon}}$ is positive definite and $\gamma>0$, the speculative component of a trader's demand in an asset $k$ is decreasing in its own price for every asset $k$. His "market making" aggressiveness is captured by the difference between traders' total net demand and informed agents' speculative aggressiveness in each asset for a unitary price vector. This matrix has no particular structure and thus nothing can be said a priori about the sign of its diagonal elements. Indeed, as $\boldsymbol{p}$ differs from $\overline{\boldsymbol{v}}$ either because of noise traders' liquidity shocks, or because of informed traders' demands, an informed agent attempts to establish whether the order he faces is due to the former or to the latter. If $\left(\boldsymbol{\Lambda}^{-1}\right)_{k k}-\mathbf{A}_{k k}>0$, then he attributes it to a supply shock and thus accommodates 
it. 4 This corresponds to the "normal goods" case of consumer theory in which the cheaper is an asset, the more of it a trader wants to buy. If, however, $\left(\boldsymbol{\Lambda}^{-1}\right)_{k k}-\mathbf{A}_{k k} \leq 0$, then the trader attributes the total net demand he faces to informed trading, refrains from taking the other side of the trade, and a Giffen good may arise.

Notice that Giffen goods in the present context have a different interpretation from the one they have in consumer theory. Indeed, in the latter setting prices are exogenous to traders' demands, whereas in the former prices are endogenous equilibrium prices and demand functions are equilibrium demands. Furthermore, a Giffen good in consumer theory is due to the presence of a strong income effect that offsets the substitution effect and leads to an increase (decrease) in the trader's demand when the good's price increases (decreases). However, in the current setting, owing to the assumed exponential utility function and the presence of a riskless asset, income effects do not exist. As the following examples show, an asset here can be a Giffen good as a result of the information extraction problem that informed traders face when forming the market making component of their demand functions.

Example 1 Suppose $K=2$ and indicate with $\tau_{x_{k}}$ and $\rho_{x}$, respectively the precision of the random variable $x_{k}$ and the correlation coefficient of the random variables $x_{1}, x_{2}$. Suppose $\rho_{v}=\rho_{\epsilon}=\rho_{u}=0$, then

$$
\mathbf{A}=\gamma\left(\begin{array}{cc}
\tau_{\epsilon_{1}} & 0 \\
0 & \tau_{\epsilon_{2}}
\end{array}\right), \quad \boldsymbol{\Lambda}=\left(\begin{array}{cc}
\lambda_{1} & 0 \\
0 & \lambda_{2}
\end{array}\right)
$$

where $\lambda_{k}=\left(1+\gamma a_{k} \tau_{u_{k}}\right) /\left(a_{k}+\gamma \tau_{k}\right), a_{k}=\mathbf{A}_{k k}=\gamma \tau_{\epsilon_{k}}$, and $\tau_{k}=\tau_{v_{k}}+a_{k}^{2} \tau_{u_{k}}$ indicate, respectively, the reciprocal of market depth and the public precision associated with market $k=1,2$. Hence, traders' strategies are given by

$$
X_{i k}\left(\boldsymbol{s}_{i}, \boldsymbol{p}\right)=a_{k}\left(s_{i k}-p_{k}\right)+\frac{\gamma \tau_{v_{k}}}{1+\gamma \tau_{u_{k}} a_{k}}\left(\bar{v}_{k}-p_{k}\right)
$$

As explained above, informed traders have two trading motives: they speculate on private information, and they absorb the supply shock taking the counterpart of the aggregate order in each asset and clearing markets (i.e. buying when the price declines and selling when it increases w.r.t. its expected value). While speculation is due to private information, "market making" is the result of the price discount (premium) informed traders receive on each

\footnotetext{
${ }^{4}$ To be sure: $\boldsymbol{\Lambda}^{-1}$ and A respectively measure the size of total traders' demand and the speculative component of each informed trader's demand for a unitary price vector. Therefore if $\left(\boldsymbol{\Lambda}^{-1}\right)_{k k}-\mathbf{A}_{k k}>0$, $\left(\boldsymbol{\Lambda}^{-1}\right)_{k k}-\mathbf{A}_{k k}$ captures the part of the total net demand for asset $k$ that for a unitary price vector, in the trader's opinion, is not due to informed agents' superior information about asset $k$.
} 
transaction because of risk aversion. To see this, rewrite prices and strategies as follows:

$$
\begin{aligned}
p_{k} & =E\left[v_{k} \mid z_{k}\right]-\frac{\tau_{v_{k}}}{a_{k} \tau_{u_{k}}\left(a_{k}+\gamma \tau_{k}\right)}\left(\bar{v}_{k}-E\left[v_{k} \mid z_{k}\right]\right), \\
X_{i k}\left(\boldsymbol{s}_{i}, \boldsymbol{p}\right) & =a_{k}\left(s_{i k}-p_{k}\right)+\frac{\gamma \tau_{k} \tau_{v_{k}}}{a_{k} \tau_{u_{k}}\left(a_{k}+\gamma \tau_{k}\right)}\left(\bar{v}_{k}-E\left[v_{k} \mid z_{k}\right]\right) .
\end{aligned}
$$

Whenever the traders in the market for asset $k$ believe that on average asset $k$ 's value is lower than its unconditional expectation (i.e. $\bar{v}_{k}>E\left[v_{k} \mid z_{k}\right]$ ) an informed trader buys the asset at a discount (i.e. $\left.p_{k}-E\left[v_{k} \mid z_{k}\right]\right)$ to be compensated for the risk that $v_{k}<E\left[v_{k} \mid z_{k}\right]$. The opposite happens when traders on average believe the asset value to be higher than its unconditional expected value: in this case a trader sells the asset at a premium.

Clearly $a_{k}=\gamma \tau_{\epsilon_{k}}>0$ and $\left(\tau_{v_{k}} /\left(1+\gamma \tau_{u_{k}} a_{k}\right)\right)>0$. Thus, no Giffen good appears in this case.

Example 2 Suppose now, as in Admati (1985), that $\mathbf{A}=I$ and

$$
\boldsymbol{\Pi}_{\boldsymbol{v}}^{-1}=\left(\begin{array}{cc}
1 & 5 \\
5 & 26
\end{array}\right), \quad \Pi_{\boldsymbol{u}}^{-1}=\left(\begin{array}{ll}
5 & 3 \\
3 & 2
\end{array}\right)
$$

Then

$$
\boldsymbol{\Lambda}=\left(\begin{array}{ll}
-0.02 & 0.19 \\
-0.45 & 1.08
\end{array}\right), \quad \mathbf{\Lambda}^{-1}-\mathbf{A}=\left(\begin{array}{cc}
15.6 & -3 \\
7 & -1.3
\end{array}\right)
$$

and traders' strategies are given by:

$$
\begin{aligned}
& X_{i 1}\left(\boldsymbol{s}_{i}, \boldsymbol{p}\right)=s_{i 1}-p_{1}+15.6\left(\bar{v}_{1}-p_{1}\right)-3\left(\bar{v}_{2}-p_{2}\right), \\
& X_{i 2}\left(\boldsymbol{s}_{i}, \boldsymbol{p}\right)=s_{i 2}-p_{2}-1.3\left(\bar{v}_{2}-p_{2}\right)+7\left(\bar{v}_{1}-p_{1}\right) .
\end{aligned}
$$

Notice that asset 2 is a Giffen good: an increase (decrease) in its price leads the trader to increase (decrease) his position in the asset. Furthermore, notice that in (2.2) the Giffen good "anomaly" is entirely due to the market making component of the trader's demand. In particular, whenever $\bar{v}_{2}>p_{2}$, traders are no longer willing to accommodate the supply shock (as in example 1). Rather, for any given value of the speculative component of their demand, in the presence of a price decrease they reduce their position in the asset. This is so because of the information they extract from the observation of the aggregate orders in the two assets. Indeed, suppose $\bar{v}_{1}>p_{1}$ and $\bar{v}_{2}>p_{2}$. Should traders attribute this price realization to informed trading or to a supply shock? The positive correlation across pay-offs makes a contemporaneous value reduction in both assets likely. However, as the distribution of asset 1 is more concentrated than the one of asset $2, \bar{v}_{1}>p_{1}$ is probably due to a selling pressure from noise traders; on the contrary $\bar{v}_{2}>p_{2}$ may be the result of "bad news." Such inference is 
reinforced by the higher dispersion of noise traders' demand in asset 1 (w.r.t. asset 2) and by the fact that noise traders' demands are positively correlated. Hence, informed traders align their behavior to the rest of the market in asset 2 and "lean against the wind" in asset 1.

Expressing the equilibrium price and a trader's demand as done in example 1 sheds further light on the market making component. Indeed, rearranging (2.1) gives:

$$
\begin{aligned}
\boldsymbol{p} & =E[\boldsymbol{v} \mid \boldsymbol{z}]-\left(\mathbf{A} \boldsymbol{\Pi}_{\boldsymbol{u}}(\mathbf{A}+\gamma \boldsymbol{\Pi})\right)^{-1} \boldsymbol{\Pi}_{\boldsymbol{v}}(\overline{\boldsymbol{v}}-E[\boldsymbol{v} \mid \boldsymbol{z}]) \\
\mathbf{X}_{i}\left(\boldsymbol{s}_{i}, \boldsymbol{p}\right) & =\mathbf{A}\left(\boldsymbol{s}_{i}-\boldsymbol{p}\right)+\gamma \boldsymbol{\Pi}\left(\mathbf{A} \boldsymbol{\Pi}_{\boldsymbol{u}}(\mathbf{A}+\gamma \boldsymbol{\Pi})\right)^{-1} \boldsymbol{\Pi}_{\boldsymbol{v}}(\overline{\boldsymbol{v}}-E[\boldsymbol{v} \mid \boldsymbol{z}])
\end{aligned}
$$

Using the above parameter values:

$$
\begin{aligned}
& p_{1}=E\left[v_{1} \mid \boldsymbol{z}\right]-9.7\left(\bar{v}_{1}-E\left[v_{1} \mid \boldsymbol{z}\right]\right)+1.85\left(\bar{v}_{2}-E\left[v_{2} \mid \boldsymbol{z}\right]\right), \\
& p_{2}=E\left[v_{2} \mid \boldsymbol{z}\right]-20.8\left(\bar{v}_{1}-E\left[v_{1} \mid \boldsymbol{z}\right]\right)+3.98\left(\bar{v}_{2}-E\left[v_{2} \mid \boldsymbol{z}\right]\right),
\end{aligned}
$$

and,

$$
\begin{aligned}
& X_{i 1}\left(\boldsymbol{s}_{i}, \boldsymbol{p}\right)=s_{i 1}-p_{1}+105.3\left(\bar{v}_{1}-E\left[v_{1} \mid \boldsymbol{z}\right]\right)-20.14\left(\bar{v}_{2}-E\left[v_{2} \mid \boldsymbol{z}\right]\right), \\
& X_{i 2}\left(\boldsymbol{s}_{i}, \boldsymbol{p}\right)=s_{i 2}-p_{2}+47.19\left(\bar{v}_{1}-E\left[v_{1} \mid \boldsymbol{z}\right]\right)-9.02\left(\bar{v}_{2}-E\left[v_{2} \mid \boldsymbol{z}\right]\right) .
\end{aligned}
$$

Notice that differently from example 1, a trader is not willing to accommodate the total net demand in asset 2 . Whenever the traders in the market for asset 2 believe that on average asset 2's value is lower than its unconditional expectation $\left(\bar{v}_{2}>E\left[v_{2} \mid \boldsymbol{z}\right]\right)$, an informed trader sells the asset at a premium (instead of buying it at a discount) to be compensated for the risk that $v_{2}>E\left[v_{2} \mid \boldsymbol{z}\right]$.

Summarizing, when all traders in the market are risk averse, the demand of an informed agent can be decomposed into a speculative and a market making component. Owing to correlation effects, the market making component may make informed agents willing to increase (decrease) their position in a given asset when its price increases (decreases). Thus, intuitively, if an informed agent were to find it unprofitable to accommodate the total net demand, the market making component should disappear rendering his demand well behaved in prices. ${ }^{6}$ The next section shows that this intuition is indeed correct.

\footnotetext{
${ }^{5}$ Strictly speaking, the trader decumulates his long position if $s_{i 2}-p_{2}>0$ and accumulates it if the reverse happens.

${ }^{6}$ It is important to emphasize, though, that such a decomposition is based on the trader's private information. As traders' information is diverse, what a trader thinks of being a non-information-driven trade may be perceived as information-driven by another trader (see He and Wang (1995) for a discussion of this issue in the context of a one-asset, dynamic, noisy rational expectations equilibrium model).
} 


\section{The Market with Risk Neutral Market Makers}

In this section I keep the same information structure of section 2 and introduce competitive risk neutral market makers as in Vives (1995a) and Cespa (2001). Market makers can be seen as uninformed agents that aggregate all traders' orders and set a single market clearing price vector. As a result of risk neutrality, prices do not incorporate a risk premium and informed traders find it unprofitable to accommodate traders' total orders. Hence, they only trade to speculate on private information and their demand functions are well behaved. However, market makers, insofar as clear all trades, use the equilibrium price to disentangle noise from information and correlation effects can induce the Giffen phenomenon in their demand functions.

More formally, let each informed trader $i$ submit a vector of demand functions $\mathbf{X}_{I i}\left(\boldsymbol{s}_{i}, \boldsymbol{p}\right)$, indicating the position desired in each asset $k$ at every price vector $\boldsymbol{p}$, contingent on his private information. Noise traders' demand $\boldsymbol{u}$ is price inelastic and random. Risk neutral market makers observe the aggregate order flow $\mathbf{L}(\cdot)=\int_{0}^{1} \mathbf{X}_{I i} d i+\boldsymbol{u}$ and set prices efficiently: $\boldsymbol{p}=E[\boldsymbol{v} \mid \boldsymbol{p}] .{ }^{7}$ Restricting attention to linear equilibria, the following result holds:

Proposition 2 In the market with competitive, risk neutral market makers, there exists a unique linear equilibrium where informed traders $(I)$ and market makers $(M M)$ trade according to the following functions:

$$
\begin{aligned}
& \mathbf{X}_{I i}\left(\boldsymbol{s}_{i}, \boldsymbol{p}\right)=\mathbf{A}\left(\boldsymbol{s}_{i}-\boldsymbol{p}\right) \\
& \mathbf{X}_{M M j}(\boldsymbol{p})=\left(\boldsymbol{\Lambda}_{R N}^{-1}-\mathbf{A}\right)(\overline{\boldsymbol{v}}-\boldsymbol{p}),
\end{aligned}
$$

and prices are given by $\boldsymbol{p}=E[\boldsymbol{v} \mid \boldsymbol{z}]=\boldsymbol{\Lambda}_{R N} \boldsymbol{z}+\left(I-\boldsymbol{\Lambda}_{R N} \mathbf{A}\right) \overline{\boldsymbol{v}}$, where $\mathbf{A}=\gamma \boldsymbol{\Pi}_{\boldsymbol{\epsilon}}, \boldsymbol{z}=\mathbf{A} \boldsymbol{v}+\boldsymbol{u}$, $\boldsymbol{\Lambda}_{R N}=\boldsymbol{\Pi}^{-1} \mathbf{A} \boldsymbol{\Pi}_{\boldsymbol{u}}$, and $\boldsymbol{\Pi}=(\operatorname{Var}[\boldsymbol{v} \mid \boldsymbol{z}])^{-1}=\boldsymbol{\Pi}_{\boldsymbol{v}}+\mathbf{A} \boldsymbol{\Pi}_{\boldsymbol{u}} \mathbf{A}$.

Proof. See the appendix.

Remark 1 Notice that as the matrix of traders' speculative aggressiveness (A) coincides in propositions 1 and 2, the informational content of the order flows $(\boldsymbol{z})$ does not change in the two equilibria. As a consequence, the inference traders can make by observing equilibrium prices in the two markets is the same.

\footnotetext{
${ }^{7}$ As will become clear in the proof of proposition 2 , in equilibrium $\boldsymbol{p}$ is observationally equivalent to $\boldsymbol{z}$. Therefore, $\boldsymbol{p}=E[\boldsymbol{v} \mid \boldsymbol{z}]=E[\boldsymbol{v} \mid \boldsymbol{p}]$. Efficient pricing can be seen as the result of Bertrand competition among risk neutral market makers for each asset order flow (see Vives (1995b)).
} 
Informed traders' behavior has now changed. Owing to market makers' risk neutrality, the risk premia incorporated into asset prices disappear and market making becomes unprofitable to risk-averse, informed traders. Therefore, as $\mathbf{A}$ is positive definite, no Giffen good appears in their demand functions. On the contrary, market makers' demand may still display the anomaly as the following example shows.

Example 3 Keeping the data of example 2, $\mathbf{A}=I$ and

$$
\boldsymbol{\Lambda}_{R N}=\left(\begin{array}{ll}
-0.11 & 0.21 \\
-0.65 & 1.11
\end{array}\right), \quad \boldsymbol{\Lambda}_{R N}^{-1}-\mathbf{A}=\left(\begin{array}{cc}
115 & -22 \\
68 & -13
\end{array}\right) .
$$

Hence, $X_{I i, 1}\left(\boldsymbol{s}_{i}, \boldsymbol{p}\right)=s_{i 1}-p_{1}, X_{I i, 2}\left(\boldsymbol{s}_{i}, \boldsymbol{p}\right)=s_{i 2}-p_{2}, X_{M M j, 1}(\boldsymbol{p})=115\left(\bar{v}_{1}-p_{1}\right)-22\left(\bar{v}_{2}-p_{2}\right)$, and $X_{M M j, 2}(\boldsymbol{p})=-13\left(\bar{v}_{2}-p_{2}\right)+68\left(\bar{v}_{1}-p_{1}\right)$. Asset 2 is the Giffen good and an intuition along the lines given in example 2 applies here too.

Therefore, combining the intuition drawn from examples 2 and 3 with proposition 2 and remark 1, Giffen goods cannot be the result of an information effect that "overwhelms the substitution effect" as argued by Admati (1985). If such an information effect was at the root of the anomaly, it should have also affected the strategy of an informed trader displayed in proposition 2 .

The Giffen goods anomaly comes from the role that prices perform in informed traders' strategies. With no risk neutral market makers, prices have two roles: (1) they allow to disentangle error terms from information in their private signals; (2) they allow to separate noise from information in the observed order flow realizations. The first role is related to the speculative component of the trader's demand; the second role is related to the market making component. Indeed, for $\rho_{v}, \rho_{u}, \rho_{\epsilon} \neq 0$, when $K=2$, an informed strategy is given by

$$
\begin{aligned}
& X_{i k}\left(\boldsymbol{s}_{i}, \boldsymbol{p}\right)= \\
& \quad \frac{\gamma \tau_{\epsilon_{k}}}{1-\rho_{\epsilon}^{2}}\left(s_{i k}-p_{k}\right)-\frac{\gamma \rho_{\epsilon} \sqrt{\tau_{\epsilon_{k}} \tau_{\epsilon_{h}}}}{1-\rho_{\epsilon}^{2}}\left(s_{i h}-p_{h}\right)+\sum_{l=1}^{2}\left(\boldsymbol{\Lambda}^{-1}-\mathbf{A}\right)_{k l}(\overline{\boldsymbol{v}}-\boldsymbol{p})_{k l} .
\end{aligned}
$$

To see how prices perform the first role, assume that $\rho_{\epsilon}>0$ and that trader $i$ receives two signals $s_{i k}, s_{i h}$ such that $s_{i k}>p_{k}$ and $s_{i h}>p_{h}$. This can happen for two reasons: either both assets are worth more than what the market thinks (i.e. asset prices are biased downward e.g. by noise traders' selling pressure), or both signals are biased upward. The existence of positive correlation across signal-error terms strengthens the hypothesis of a contemporaneous, upward bias into the trader's signals. ${ }^{8}$ Given this, he reinforces his belief that the good news he received is due to the effect of error biases and reduces his demand for both assets.

\footnotetext{
${ }^{8}$ This is the case because an error that biases upward the information contained in $s_{i k}$ is more likely to happen together with an error biasing upwards the information about asset $h$ as well.
} 
As far as the second role, example 2 provided an intuition for it. As soon as risk neutral market makers are introduced in the model, informed traders no longer find it profitable to absorb the liquidity shock and prices cease to perform the second role for them. However, since market makers take the counterpart of the aggregate order in each asset and clear markets, such a second role is relevant to their objectives. Hence, the Giffen goods anomaly only characterizes risk neutral market makers' demand functions.

The above conclusion also clarifies the effect of assuming infinitely dispersed noise traders' demands (see Admati (1985), p. 647). In this situation, informed traders refrain from using prices to disentangle noise from information in the observed order flows. Formally, letting $\boldsymbol{\Pi}_{\boldsymbol{u}} \rightarrow 0$ in the equilibrium of proposition 1 (in any norm on matrices) gives:

Corollary 1 In the market with no risk neutral market makers, when noise traders' demand dispersion increases without bound: $\boldsymbol{\Lambda} \rightarrow \boldsymbol{\Lambda}^{*}=(\gamma \boldsymbol{\Pi} \boldsymbol{v}+\mathbf{A})^{-1}, E[\boldsymbol{v} \mid \boldsymbol{z}] \rightarrow \overline{\boldsymbol{v}}$, and

$$
\begin{aligned}
\boldsymbol{p} & \rightarrow \boldsymbol{p}^{*}=\boldsymbol{\Lambda}^{*} \boldsymbol{z}+\left(I-\boldsymbol{\Lambda}^{*} \mathbf{A}\right) \overline{\boldsymbol{v}} \\
\mathbf{X}_{i}\left(\boldsymbol{s}_{i}, \boldsymbol{p}\right) & \rightarrow \mathbf{X}_{i}^{*}\left(\boldsymbol{s}_{i}, \boldsymbol{p}^{*}\right)=\mathbf{A}\left(\boldsymbol{s}_{i}-\boldsymbol{p}^{*}\right)+\gamma \boldsymbol{\Pi}_{\boldsymbol{v}}\left(\overline{\boldsymbol{v}}-\boldsymbol{p}^{*}\right)
\end{aligned}
$$

almost surely.

As $\boldsymbol{\Pi}_{\boldsymbol{v}}$ is positive definite and $\gamma>0$, the market making component of an informed trader's demand is well behaved and Giffen goods disappear. Indeed, as noise traders' demand dispersion increases without bound, informed traders cannot use prices to disentangle noise from information in the observed order flows realization. Furthermore, the risk of trading with an informed agent vanishes and risk averse traders are always willing to accommodate the total net demand they face at a premium. To see this, express the equilibrium price as follows:

$$
\boldsymbol{p}^{*}=\overline{\boldsymbol{v}}+\left(\gamma \boldsymbol{\Pi}_{\boldsymbol{v}}+\mathbf{A}\right)^{-1}(\boldsymbol{z}-E[\boldsymbol{z}])
$$

Notice that $\left(\gamma \boldsymbol{\Pi}_{\boldsymbol{v}}+\mathbf{A}\right)^{-1}$ is a symmetric, positive definite matrix. Hence, whenever the total net demand in an asset $k$ is higher than expected $\left(z_{k}>E\left[z_{k}\right]\right)$, an informed trader attributes the demand realization to noise traders' liquidity needs. As a consequence, he accommodates it selling the asset at a premium which is proportional to the demand "surprise." Thus, prices aggregate information (i.e. reflect the value of $\boldsymbol{z}$ ) allowing informed traders to use them to disentangle the error terms affecting their signals. Therefore, in this Walrasian equilibrium, prices perform the first role but not the second role. ${ }^{9}$

\footnotetext{
${ }^{9}$ It is interesting to contrast this equilibrium with its counterpart in the market with risk neutral market makers. As shown in proposition $[2$, the equilibrium price there is given by $\boldsymbol{p}=E[\boldsymbol{v} \mid \boldsymbol{z}]$. However, as noise
} 
Remark 2 The result that the Giffen goods anomaly only characterizes market makers' demand functions is likely to depend on the competitive assumption about informed traders' conduct, and on their risk-averse preferences. Indeed, a "strategic" insider can exploit such anomalous market-making behavior and induce a price increase to speculate on it. The latter effect is stronger, the less risk-averse the insider is, as a very risk-tolerant insider does not suffer from the unpredictability of the asset pay-offs, and thus exploits his information advantage more aggressively. This intuition leads to conjecture that in the presence of a risk-neutral, non atomistic trader, the Giffen goods anomaly should disappear also from market makers' strategies. Indeed, Caballé and Krishnan (1992) in a multi-asset generalization of Kyle (1985), find that in equilibrium the matrix mapping order flows into prices must be symmetric and positive definite, ruling out the existence of Giffen goods. 10

\section{Model's Generalizations}

In this section I consider two generalizations of the model studied in section 2: first, I add a sector of risk-averse uninformed traders to the market considered in section 2. Next, I relax the simplifying assumptions introduced in section 2. The former generalization allows to obtain a model where the equilibrium of proposition 2 arises as a limit result when the risk-bearing capacity of uninformed traders grows without bound. The latter enables to show that the intuitions so far obtained, apply to Admati's equilibrium as well.

\subsection{The Market with Uninformed Traders}

Formally, assume that a continuum of uninformed traders distributed in the interval $[0,1]$ is added to the market of section 2. Every uninformed trader $j$ 's preferences are represented by a CARA utility $U\left(\pi_{U j}\right)=-\exp \left\{-\pi_{U j} / \gamma_{U}\right\}$ where $\gamma_{U}>0$ is the coefficient of constant absolute risk tolerance and $\pi_{U j}=\boldsymbol{x}_{U j}^{\prime}(\boldsymbol{v}-\boldsymbol{p})$ is the profit of buying $\boldsymbol{x}_{U j}^{\prime}=\left(x_{U j, 1}, x_{U j, 2}, \ldots, x_{U j, K}\right)$ units of each asset at price $\boldsymbol{p}$. Assume that every uninformed trader submits a vector of

traders' demand is infinitely dispersed, market makers cannot extract any information from $\boldsymbol{z}$ to estimate $\boldsymbol{v}$. As a consequence, $\boldsymbol{p}=\overline{\boldsymbol{v}}$, informed traders cannot use the information conveyed by $\boldsymbol{z}$ to disentangle the error terms in their private signals and $\mathbf{X}_{I i}^{*}=\mathbf{A}\left(\boldsymbol{s}_{i}-\overline{\boldsymbol{v}}\right)$. Thus, differently from the case analyzed above, the presence of competitive, risk neutral market makers prevents the equilibrium price from aggregating any information about the asset payoffs.

${ }^{10}$ Being a generalization of Kyle (1985), the insider in Caballé and Krishnan's model submits non pricecontingent orders to competitive, risk-neutral market makers, differently from the informed traders of the present context. It is important to emphasize that strategic behavior per se does not prevent Giffen goods from arising in equilibrium. As Bhattacharya, Reny, and Spiegel (1995) show, in a multi-asset model where a risk-averse insider has both a "hedging" and an "informational" trading motive, the uninformed agents' demand function can still display the Giffen anomaly. 
demand functions $\mathbf{X}_{U j}(\boldsymbol{p})$ indicating the desired position in each asset $k$ at every price vector $\boldsymbol{p}$. Normalize his initial wealth to zero and indicate with $\gamma_{I}$ the risk tolerance coefficient of an informed trader. Restricting attention to linear equilibria, the following result applies:

Proposition 3 In the market with a sector of (CARA) uninformed traders $(U)$, there exists a unique linear equilibrium where agents trade according to the functions

$$
\begin{aligned}
\mathbf{X}_{I i}\left(\boldsymbol{s}_{i}, \boldsymbol{p}\right) & =\mathbf{A}\left(\boldsymbol{s}_{i}-\boldsymbol{p}\right)+\left(\gamma_{I} / \gamma\right)\left(\boldsymbol{\Lambda}_{U}^{-1}-\mathbf{A}\right)(\overline{\boldsymbol{v}}-\boldsymbol{p}) \\
\mathbf{X}_{U j}(\boldsymbol{p}) & =\left(\gamma_{U} / \gamma\right)\left(\boldsymbol{\Lambda}_{U}^{-1}-\mathbf{A}\right)(\overline{\boldsymbol{v}}-\boldsymbol{p})
\end{aligned}
$$

and prices are given by $\boldsymbol{p}=\boldsymbol{\Lambda}_{U} \boldsymbol{z}+\left(I-\boldsymbol{\Lambda}_{U} \mathbf{A}\right) \overline{\boldsymbol{v}}$, where $\mathbf{A}=\gamma_{I} \boldsymbol{\Pi}_{\boldsymbol{\epsilon}}, \boldsymbol{z}=\mathbf{A} \boldsymbol{v}+\boldsymbol{u}, \boldsymbol{\Lambda}_{U}=$ $(\mathbf{A}+\gamma \boldsymbol{\Pi})^{-1}(I+\gamma \mathbf{A} \Pi \boldsymbol{u}), \boldsymbol{\Pi}=(\operatorname{Var}[\boldsymbol{v} \mid \boldsymbol{z}])^{-1}=\boldsymbol{\Pi}_{\boldsymbol{v}}+\mathbf{A} \boldsymbol{\Pi} \boldsymbol{u} \mathbf{A}$, and $\gamma=\gamma_{I}+\gamma_{U}$.

Proof. See the appendix.

QED

Notice that informed traders speculate on private information (as in proposition 1) and, together with uninformed traders, accommodate the total net demand. As in proposition 1, the Giffen goods anomaly (potentially) comes from the market making component of a trader's demand.

Corollary 2 If $\gamma_{U} \rightarrow \infty$ the equilibrium of proposition 3 converges (almost surely) to the one of proposition 2 .

Proof. It follows immediately from the fact that $\mathbf{A}$ does not depend on $\gamma_{U}$ and as $\gamma_{U} \rightarrow \infty$, $\boldsymbol{\Lambda}_{U} \rightarrow \boldsymbol{\Lambda}_{R N}$. Thus, $\boldsymbol{p}$ converges (almost surely) to $E[\boldsymbol{v} \mid \boldsymbol{z}]$.

QED

Therefore, as the aggregate risk-bearing capacity of uninformed traders increases, the risk premia incorporated into equilibrium prices disappear, informed traders find no longer profitable to accommodate the total net demand and their demand function becomes "well behaved" in prices.

\subsection{The Model with Non-Identically Distributed Signals}

One may wonder about what is the effect of the simplifying assumptions on the information structure made in section 2 . In particular, is the demand decomposition obtained in proposition 11 due to the fact that traders' signals are i.i.d.? If this was the case, then the Giffen goods anomaly may not only be due to the market making component of traders' demand and the analysis of the previous sections would only apply to a particular category of noisy rational expectations linear equilibria with multiple assets. However, it turns out that the 
results are general and that one can apply the same decomposition also to the market studied by Admati (1985).

In particular, let us modify the informational assumptions of section 2.1 in the following two aspects:

A1. Traders' private information, risk tolerance and initial wealth. Traders receive a vector of private signals of a potentially different conditional precision: $\boldsymbol{s}_{i}=\boldsymbol{v}+\boldsymbol{\epsilon}_{i}$ is such that $\boldsymbol{\epsilon}_{i} \sim N\left(\mathbf{0}, \boldsymbol{\Pi}_{\boldsymbol{\epsilon}_{i}}^{-1}\right), \boldsymbol{\epsilon}_{i}$ and $\boldsymbol{\epsilon}_{j}$ are independent for $i \neq j, \boldsymbol{\Pi}_{\boldsymbol{\epsilon}_{i}}^{-1}$ is positive definite for every $i$, and $\boldsymbol{\Pi}_{\boldsymbol{\epsilon}_{i}}^{-1} \neq \boldsymbol{\Pi}_{\boldsymbol{\epsilon}_{j}}^{-1}$ for $i \neq j$; risk tolerance coefficients are different across traders: $\gamma_{i} \neq \gamma_{j}$ for $i \neq j$; finally, a trader $i$ 's initial wealth is $W_{0 i}>0$.

A2. Noise traders' demand and the risk free asset return. Noise traders' expected demand is non null: $\boldsymbol{u} \sim N\left(\overline{\boldsymbol{u}}, \boldsymbol{\Pi}_{\boldsymbol{u}}^{-1}\right)$; and the return on the risk free asset is given by $R \geq 1$.

With the above assumptions the model outlined in section 2 coincides with the one studied by Admati (1985). Therefore, the following result holds:

Proposition 4 In the market outlined in section 2 enriched with the assumptions $A 1$ and $A 2$, there exists a unique linear equilibrium where informed agents trade according to the function

$$
\begin{aligned}
& \mathbf{X}_{i}\left(\boldsymbol{s}_{i}, \boldsymbol{p}\right)= \\
& \quad \mathbf{A}_{i}\left(\boldsymbol{s}_{i}-R \boldsymbol{p}\right)+\left(\gamma_{i} / \bar{\gamma}\right)\left(\overline{\boldsymbol{\Lambda}}^{-1}-\overline{\mathbf{A}}\right)(\overline{\boldsymbol{v}}-R \boldsymbol{p})+\left(\gamma_{i} / \bar{\gamma}\right)\left(I+\bar{\gamma} \overline{\mathbf{A}} \Pi_{\boldsymbol{u}}\right)^{-1} \overline{\mathbf{A}} \Pi_{\boldsymbol{u}} \overline{\boldsymbol{u}},
\end{aligned}
$$

and the vector of equilibrium prices is given by

$$
\boldsymbol{p}=(1 / R) \overline{\boldsymbol{\Lambda}} \boldsymbol{z}+(1 / R)(I-\overline{\mathbf{\Lambda}} \overline{\mathbf{A}}) \overline{\boldsymbol{v}}+(\bar{\gamma} / R)\left(\bar{\gamma}\left(\boldsymbol{\Pi}_{\boldsymbol{v}}+\overline{\mathbf{A}} \Pi_{\boldsymbol{u}} \overline{\mathbf{A}}\right)+\overline{\mathbf{A}}\right)^{-1} \overline{\mathbf{A}} \Pi_{\boldsymbol{u}} \overline{\boldsymbol{u}}
$$

where $\mathbf{A}_{i}=\gamma_{i} \boldsymbol{\Pi}_{\boldsymbol{\epsilon}_{i}}, \bar{\gamma}=\int_{0}^{1} \gamma_{i} d i, \overline{\mathbf{A}}=\int_{0}^{1} \gamma_{i} \boldsymbol{\Pi}_{\boldsymbol{\epsilon}_{i}} d i, \boldsymbol{z}=\overline{\mathbf{A}} \boldsymbol{v}+\boldsymbol{u}$, and $\overline{\boldsymbol{\Lambda}}=\left(\bar{\gamma}\left(\boldsymbol{\Pi}_{\boldsymbol{v}}+\overline{\mathbf{A}} \boldsymbol{\Pi} \boldsymbol{u} \overline{\mathbf{A}}\right)+\right.$ $\overline{\mathbf{A}})^{-1}\left(I+\bar{\gamma} \overline{\mathbf{A}} \Pi_{\boldsymbol{u}}\right)$.

Proof. See Admati (1985).

QED

As one can verify according to (4.3), a trader's strategy has both a "speculative" and a "market making" component as (2.1). As $\mathbf{A}_{i}=\gamma_{i} \boldsymbol{\Pi}_{\boldsymbol{\epsilon}_{i}}, \gamma_{i}>0$ and $\boldsymbol{\Pi}_{\boldsymbol{\epsilon}_{i}}$ is positive definite for all $i$, Giffen goods can only be due to the "market making" component. Notice also that as $E[\boldsymbol{u}]=\overline{\boldsymbol{u}}$, informed speculators expect to accommodate a constant supply shock $\overline{\boldsymbol{u}}$. Thus, owing to the presence of a non-null expected noise traders' demand, the market making component in this equilibrium reflects both realized and expected factors.

If $\gamma_{i}=\gamma$ and $\boldsymbol{\Pi}_{\boldsymbol{\epsilon}_{i}}=\boldsymbol{\Pi}_{\boldsymbol{\epsilon}}$ for all $i, \overline{\boldsymbol{u}}=\mathbf{0}$ and $R=1$, traders' speculative aggressiveness coincides for all agents and is given by $\mathbf{A}=\gamma \boldsymbol{\Pi}_{\boldsymbol{\epsilon}}$, the "expected" market making component in (4.3) vanishes and the equilibrium of proposition 4 coincides with the one of proposition 1. 


\section{Conclusions}

Recent work in finance theory has highlighted the role played by Giffen goods in affecting stock market behavior around "unusual" events. Giffen goods characterize both unstable equilibria and episodes of market crashes. Indeed, when faced with the problem of extracting a signal about the asset fundamentals from the observed aggregate orders, traders with a "backward bending" demand curve shy away from assets whose price plummets and increase their position in assets whose price rockets. Depending on the specific model, this either destabilizes the market (as in Cespa (2002)) or introduces discontinuities in the function mapping the asset supply into its equilibrium price (as in Gennotte and Leland (1990) and Barlevy and Veronesi (2002)). These contributions testify the importance of understanding the conditions under which Giffen goods arise in markets with asymmetric information.

Building on the result of Admati (1985), in this paper I have shown that contrary to previous intuitions in a market where informed and noise traders exchange vectors of assets, information effects per se are not responsible for the existence of Giffen goods. The role that prices play in informed traders' strategies also matters. In particular, I have demonstrated that whenever all agents in a market are risk averse, an informed trader has two trading motives: speculation and market making. Insofar as the trader uses equilibrium prices to separate informed from noise traders' orders, the presence of correlation effects can lead him to attribute the total net demand he faces to informed trading. As a consequence, he may thus refrain from taking the other side of the trade, giving rise to the Giffen goods anomaly. I have then given sufficient conditions that allow to remove the anomaly from informed traders demands.

While the demand decomposition result is robust to general model specifications, ${ }^{11}$ the analysis clearly relies on the assumptions about informed traders' behavior and preferences. Indeed, as conjectured in the paper, the presence of a risk-neutral, non-atomistic trader should rule out the Giffen phenomenon from all traders' strategies. In particular, it would be interesting to study a model where risk-averse, imperfectly competitive insiders submit multidimensional demand functions to risk neutral market makers. In this setup, one could analyze the behavior of the equilibrium price mapping as the insiders' risk-tolerance increases and the number of insiders grows large.

\footnotetext{
${ }^{11}$ Within the realm of the CARA-multivariate-Normal model with independent private signals.
} 


\section{Appendix}

The following lemma, which is useful to compute conditional expected values, adapts a standard result from normal theory to the present context (see e.g. DeGroot (1969), Theorem 1, section 9.9).

Lemma 1 Suppose that $\mathbf{X}_{1}, \mathbf{X}_{2}, \ldots, \mathbf{X}_{n}$ is a random sample from a multivariate normal distribution with unknown mean vector $\mathbf{M}$ and specified precision matrices $\boldsymbol{\Sigma}_{i}, i=1,2, \ldots, n$. Suppose also that the prior distribution of $\mathbf{M}$ is multivariate normal with mean vector $\boldsymbol{\mu}_{o}$ and precision matrix $\boldsymbol{\Sigma}_{o}$ such that $\boldsymbol{\mu}_{o} \in \mathbb{R}^{K}$ and $\boldsymbol{\Sigma}_{o}$ is a symmetric positive definite matrix. Then the posterior distribution of $\mathbf{M}$ when $\mathbf{X}_{i}=\mathbf{x}_{i}(i=1,2, \ldots, n)$ is a multivariate normal with mean vector $\boldsymbol{\mu}_{1}$ and precision matrix $\boldsymbol{\Sigma}_{1}=\boldsymbol{\Sigma}_{o}+\sum_{i=1}^{n} \boldsymbol{\Sigma}_{i}$, where $\boldsymbol{\mu}_{1}=\boldsymbol{\Sigma}_{1}^{-1}\left(\boldsymbol{\Sigma}_{o} \boldsymbol{\mu}_{o}+\left(\sum_{i=1}^{n} \boldsymbol{\Sigma}_{i}\right) \widetilde{\boldsymbol{x}}\right)$ and $\widetilde{\boldsymbol{x}}=\left(\sum_{i=1}^{n} \boldsymbol{\Sigma}_{i}\right)^{-1}\left(\sum_{i=1}^{n} \boldsymbol{\Sigma}_{i} \boldsymbol{x}_{i}\right)$.

Proof. For $\mathbf{M}=\boldsymbol{m}$ and $\mathbf{X}_{i}=\boldsymbol{x}_{i}(i=1,2, \ldots, n)$, the likelihood function $f_{n}\left(\boldsymbol{x}_{1}, \boldsymbol{x}_{2}, \ldots, \boldsymbol{x}_{n} \mid \boldsymbol{m}\right)$ satisfies the following relation:

$$
f_{n}\left(\boldsymbol{x}_{1}, \boldsymbol{x}_{2}, \ldots, \boldsymbol{x}_{n} \mid \boldsymbol{m}\right) \propto \exp \left\{-(1 / 2) \sum_{i=1}^{n}\left(\boldsymbol{x}_{i}-\boldsymbol{m}\right)^{\prime} \boldsymbol{\Sigma}_{i}\left(\boldsymbol{x}_{i}-\boldsymbol{m}\right)\right\} .
$$

However, it can be verified that

$$
\sum_{i=1}^{n}\left(\boldsymbol{x}_{i}-\boldsymbol{m}\right)^{\prime} \boldsymbol{\Sigma}_{i}\left(\boldsymbol{x}_{i}-\boldsymbol{m}\right)=(\boldsymbol{m}-\widetilde{\boldsymbol{x}})^{\prime}\left(\sum_{i=1}^{n} \boldsymbol{\Sigma}_{i}\right)(\boldsymbol{m}-\widetilde{\boldsymbol{x}})+\sum_{i=1}^{n}\left(\boldsymbol{x}_{i}-\widetilde{\boldsymbol{x}}\right)^{\prime} \boldsymbol{\Sigma}_{i}\left(\boldsymbol{x}_{i}-\widetilde{\boldsymbol{x}}\right) .
$$

Since the last term in the previous equation does not involve $\boldsymbol{m}$, we can rewrite (5.4) as follows:

$$
f_{n}\left(\boldsymbol{x}_{1}, \boldsymbol{x}_{2}, \ldots, \boldsymbol{x}_{n} \mid \boldsymbol{m}\right) \propto \exp \left\{-(1 / 2)(\boldsymbol{m}-\widetilde{\boldsymbol{x}})^{\prime}\left(\sum_{i=1}^{n} \boldsymbol{\Sigma}_{i}\right)(\boldsymbol{m}-\widetilde{\boldsymbol{x}})\right\} .
$$

The prior p.d.f. of $\mathbf{M}$ satisfies

$$
\varphi(\boldsymbol{m}) \propto \exp \left\{-(1 / 2)\left(\boldsymbol{m}-\boldsymbol{\mu}_{o}\right)^{\prime} \boldsymbol{\Sigma}_{o}\left(\boldsymbol{m}-\boldsymbol{\mu}_{o}\right)\right\}
$$

and the posterior p.d.f. $g\left(\cdot \mid \boldsymbol{x}_{1}, \boldsymbol{x}_{2}, \ldots, \boldsymbol{x}_{n}\right)$ of $\mathbf{M}$ will be proportional to the product of the functions specified by (5.5) and (5.6). However, one can verify that

$$
\begin{aligned}
\left(\boldsymbol{m}-\boldsymbol{\mu}_{o}\right)^{\prime} \boldsymbol{\Sigma}_{o}\left(\boldsymbol{m}-\boldsymbol{\mu}_{o}\right)+(\boldsymbol{m}-\widetilde{\boldsymbol{x}})^{\prime}\left(\sum_{i=1}^{n} \boldsymbol{\Sigma}_{i}\right)(\boldsymbol{m}-\widetilde{\boldsymbol{x}}) & =\left(\boldsymbol{m}-\boldsymbol{\mu}_{1}\right)^{\prime} \boldsymbol{\Sigma}_{1}\left(\boldsymbol{m}-\boldsymbol{\mu}_{1}\right) \\
& + \text { terms not involving } \boldsymbol{m} .
\end{aligned}
$$


Hence, we can write

$$
g\left(\boldsymbol{m} \mid \boldsymbol{x}_{1}, \boldsymbol{x}_{2}, \ldots, \boldsymbol{x}_{n}\right) \propto \exp \left\{-(1 / 2)\left(\boldsymbol{m}-\boldsymbol{\mu}_{1}\right)^{\prime} \boldsymbol{\Sigma}_{1}\left(\boldsymbol{m}-\boldsymbol{\mu}_{1}\right)\right\}
$$

The p.d.f. specified by (5.7) is that of a multivariate normal distribution for which the mean vector and the precision matrix are as specified in the statement of the lemma.

QED

Proof of proposition 2

When submitting his demand, a trader $i$ has available the vector of signals $\boldsymbol{s}_{i}$. In any linear equilibrium, due to the strong law of large numbers, private and public information are conditionally independent. Therefore, the trader's strategy depends both on $\boldsymbol{s}_{i}$, and on the equilibrium price vector $\boldsymbol{p}$. Assume the agent submits a vector of demand schedules $\mathbf{X}_{I i}\left(\boldsymbol{s}_{i}, \boldsymbol{p}\right)$, indicating the position desired in each asset $k$ at every price vector $\boldsymbol{p}$, contingent on the available information, and restrict attention to linear equilibria. Market makers thus observe the vector of aggregate order flows $\mathbf{L}(\cdot)=\int_{0}^{1} \mathbf{X}_{I i} d i+\boldsymbol{u}$.

Consider a candidate symmetric, linear equilibrium $\mathbf{X}_{I i}\left(\boldsymbol{s}_{i}, \boldsymbol{p}\right)=\mathbf{A} \boldsymbol{s}_{i}+\phi(\boldsymbol{p})$, where $\mathbf{A}$ and $\phi(\cdot)$ are respectively the matrix of trading intensities and a linear function of current prices. Also, assume for the time being that the matrix $\mathbf{A}$ is nonsingular (in equilibrium this assumption turns out to be correct). Owing to linear strategies, the aggregate order flow is then: $\mathbf{L}(\cdot)=\boldsymbol{z}+\phi(\boldsymbol{p})$, where $\boldsymbol{z}=\mathbf{A} \boldsymbol{v}+\boldsymbol{u}$, is the vector of order flows' informational contents. Because of competition for each order flow and risk neutrality, market makers set the equilibrium price equal to the conditional expectation of the pay-off vector given the informational content of the order flows. Notice that $\mathbf{A}^{-1} \boldsymbol{z} \mid \boldsymbol{v} \sim N\left(\boldsymbol{v}, \mathbf{A}^{-1} \boldsymbol{\Pi}_{\boldsymbol{u}}^{-1}\left(\mathbf{A}^{\prime}\right)^{-1}\right)$, hence we can apply lemma 1 with $n=1, \boldsymbol{m}=\boldsymbol{v}$ and $\boldsymbol{x}_{1}=\mathbf{A}^{-1} \boldsymbol{z}$ to obtain: $\boldsymbol{p}=E[\boldsymbol{v} \mid \boldsymbol{z}]$ $=\boldsymbol{\Pi}^{-1}\left(\boldsymbol{\Pi}_{\boldsymbol{v}} \overline{\boldsymbol{v}}+\mathbf{A}^{\prime} \boldsymbol{\Pi}_{\boldsymbol{u}} \boldsymbol{z}\right)=\boldsymbol{\Lambda}_{R N} \boldsymbol{z}+\left(I-\boldsymbol{\Lambda}_{R N} \mathbf{A}\right) \overline{\boldsymbol{v}}$, where $\boldsymbol{\Pi}=(\operatorname{Var}[\boldsymbol{v} \mid \boldsymbol{z}])^{-1}=\boldsymbol{\Pi}_{\boldsymbol{v}}+\mathbf{A}^{\prime} \boldsymbol{\Pi}_{\boldsymbol{u}} \mathbf{A}$ and $\boldsymbol{\Lambda}_{R N}=\boldsymbol{\Pi}^{-1} \mathbf{A}^{\prime} \Pi_{\boldsymbol{u}}$.

Given the formula for the market price I can now solve for the the demand function of a generic trader $i$. Since in equilibrium the parameters of the price are known, the trader conditions his estimation of the pay-off vector on $\boldsymbol{p}$ and $\boldsymbol{s}_{i}$. The assumption of a CARA utility function and multivariate normality gives:

$$
\mathbf{X}_{I i}\left(\boldsymbol{s}_{i}, \boldsymbol{p}\right)=\gamma\left(\operatorname{Var}\left[\boldsymbol{v} \mid \boldsymbol{s}_{i}, \boldsymbol{z}\right]\right)^{-1}\left(E\left[\boldsymbol{v} \mid \boldsymbol{s}_{i}, \boldsymbol{z}\right]-\boldsymbol{p}\right)
$$

Notice again that $\boldsymbol{s}_{i} \mid \boldsymbol{v} \sim N\left(\boldsymbol{v}, \boldsymbol{\Pi}_{\boldsymbol{\epsilon}}^{-1}\right)$ and that $\boldsymbol{p}$ in equilibrium is observationally equivalent to $\mathbf{A}^{-1} \boldsymbol{z}$ which we know satisfies $\mathbf{A}^{-1} \boldsymbol{z} \mid \boldsymbol{v} \sim N\left(\boldsymbol{v}, \mathbf{A}^{-1} \boldsymbol{\Pi}_{\boldsymbol{u}}^{-1}\left(\mathbf{A}^{\prime}\right)^{-1}\right)$. Applying again lemma 1 with $n=2, \boldsymbol{x}_{1}=\boldsymbol{s}_{i}, \boldsymbol{x}_{2}=\mathbf{A}^{-1} \boldsymbol{z}$ and $\boldsymbol{m}=\boldsymbol{v}$ gives $E\left[\boldsymbol{v} \mid \boldsymbol{s}_{i}, \boldsymbol{z}\right]=\boldsymbol{\Pi}_{i}^{-1}\left(\boldsymbol{\Pi} \boldsymbol{p}+\boldsymbol{\Pi}_{\boldsymbol{\epsilon}} \boldsymbol{s}_{i}\right)$, where $\boldsymbol{\Pi}_{i}=$ 
$\left(\operatorname{Var}\left[\boldsymbol{v} \mid \boldsymbol{s}_{i}, \boldsymbol{z}\right]\right)^{-1}=\boldsymbol{\Pi}_{\boldsymbol{v}}+\mathbf{A}^{\prime} \boldsymbol{\Pi}_{\boldsymbol{u}} \mathbf{A}+\boldsymbol{\Pi}_{\boldsymbol{\epsilon}}$. Plugging these expressions in (5.8) and simplifying, $\mathbf{X}_{I i}=\gamma \boldsymbol{\Pi}_{\boldsymbol{\epsilon}}\left(\boldsymbol{s}_{i}-\boldsymbol{p}\right)$. Identifying the demand components: $\mathbf{A}=\gamma \boldsymbol{\Pi}_{\boldsymbol{\epsilon}}$ and $\phi(\boldsymbol{p})=\mathbf{A} \boldsymbol{p}$. Notice that $\mathbf{A}=\mathbf{A}^{\prime}=\gamma \boldsymbol{\Pi}_{\boldsymbol{\epsilon}}$, hence the assumption that $\mathbf{A}$ is nonsingular is correct in equilibrium.

To determine a market maker's demand, consider the equilibrium condition

$$
\int_{0}^{1} \mathbf{X}_{I i} d i+\boldsymbol{u}+\int_{0}^{1} \mathbf{X}_{M M j} d j=\boldsymbol{z}-\mathbf{A} \boldsymbol{p}+\mathbf{X}_{M M j}=\mathbf{0} .
$$

Solving for $\boldsymbol{z}$ from the equilibrium price, substituting it in the above equation and isolating $\mathbf{X}_{M M j}(\boldsymbol{p})$ gives: $\mathbf{X}_{M M j}(\boldsymbol{p})=\left(\boldsymbol{\Lambda}_{R N}^{-1}-\mathbf{A}\right)(\overline{\boldsymbol{v}}-\boldsymbol{p})$.

QED

Proof of proposition 3

Along the same lines of the previous proof, owing to CARA utility functions and multivariate normality

$$
\begin{aligned}
\mathbf{X}_{I i}\left(\boldsymbol{s}_{i}, \boldsymbol{p}\right) & =\gamma_{I}\left(\operatorname{Var}\left[\boldsymbol{v} \mid \boldsymbol{s}_{i}, \boldsymbol{z}\right]\right)^{-1}\left(E\left[\boldsymbol{v} \mid \boldsymbol{s}_{i}, \boldsymbol{z}\right]-\boldsymbol{p}\right) \\
& =\gamma_{I} \boldsymbol{\Pi}_{\boldsymbol{\epsilon}}\left(\boldsymbol{s}_{i}-\boldsymbol{p}\right)+\gamma_{I} \boldsymbol{\Pi}(E[\boldsymbol{v} \mid \boldsymbol{z}]-\boldsymbol{p})
\end{aligned}
$$

and

$$
\begin{aligned}
\mathbf{X}_{U j}(\boldsymbol{p}) & =\gamma_{U}(\operatorname{Var}[\boldsymbol{v} \mid \boldsymbol{z}])^{-1}(E[\boldsymbol{v} \mid \boldsymbol{z}]-\boldsymbol{p}) \\
& =\gamma_{U} \boldsymbol{\Pi}(E[\boldsymbol{v} \mid \boldsymbol{z}]-\boldsymbol{p})
\end{aligned}
$$

Imposing market clearing, $\boldsymbol{p}=\boldsymbol{\Lambda}_{U} \boldsymbol{z}+\left(I-\boldsymbol{\Lambda}_{U} \mathbf{A}\right) \overline{\boldsymbol{v}}$, where $\mathbf{A}=\gamma_{I} \boldsymbol{\Pi}_{\boldsymbol{\epsilon}}, \boldsymbol{\Lambda}_{U}=(\mathbf{A}+\gamma \boldsymbol{\Pi})^{-1}(I+$ $\left.\gamma \mathbf{A} \boldsymbol{\Pi}_{\boldsymbol{u}}\right)$ and $\gamma=\gamma_{I}+\gamma_{U}$. Solving for $\boldsymbol{z}$ in the equilibrium price gives $\boldsymbol{z}=\boldsymbol{\Lambda}_{U}^{-1} \boldsymbol{p}-(I+$ $\left.\gamma \mathbf{A \Pi}_{\boldsymbol{u}}\right)^{-1} \gamma \boldsymbol{\Pi}_{\boldsymbol{v}} \overline{\boldsymbol{v}}$, and substituting it in (5.9) and (5.10) gives the demand functions for informed and uninformed traders displayed in proposition 3 .

QED

\section{References}

Admati, A. R. (1985). A noisy rational expectations equilibrium for multi-asset securities markets. Econometrica 53, 629-657.

Barlevy, G. and P. Veronesi (2002, April). Rational panics and stock market crashes. Journal of Economic Theory (forthcoming). 
Battacharya, U. and M. Spiegel (1991). Insiders, outsiders, and market breakdowns. Review of Financial Studies 4(2), 255-282.

Bhattacharya, U., P. J. Reny, and M. Spiegel (1995). Destructive interference in an imperfectly competitive multi-security market. Journal of Economic Theory 65(1), 136-170.

Caballé, J. and M. Krishnan (1992). Insider trading and asset pricing in an imperfectly competitive market. Econometrica 62, 695-704.

Cespa, G. (2001). A comparison of stock market mechanisms. UPF Working Paper 545.

Cespa, G. (2002). Short-term investment and equilibrium multiplicity. European Economic Review 46, 1645-1670.

DeGroot, M. H. (1969). Optimal Statistical Decisions. McGraw-Hill.

Diamond, D. and R. E. Verrecchia (1981). Information aggregation in a noisy rational expectations economy. Journal of Financial Economics 9, 221-235.

Gennotte, G. and H. Leland (1990). Market liquidity, hedging, and crashes. American Economic Review 80(5), 999-1021.

Grossman, S. and J. Stiglitz (1980). On the impossibility of informationally efficient markets. American Economic Review 70, 393-408.

He, H. and J. Wang (1995). Differential information and dynamic behavior of stock trading volume. Review of Financial Studies 8(4), 919-972.

Hellwig, M. F. (1980). On the aggregation of information in competitive markets. Journal of Economic Theory 22, 477-498.

Kyle, A. (1985). Continuous auctions and insider trading. Econometrica 53, 1315-1336.

Vives, X. (1995a). Short-term investment and the informational efficiency of the market. Review of Financial Studies 8(1), 125-160.

Vives, X. (1995b). The speed of information revelation in a financial market. Journal of Economic Theory 67, 178-204. 